\title{
Detection of circulating genetically abnormal cells using 4-color fluorescence in situ hybridization for the early detection of lung cancer
}

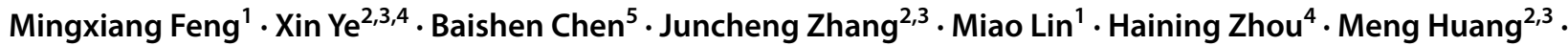 \\ Yanci Chen ${ }^{2,3} \cdot$ Yunhe Zhu ${ }^{4} \cdot$ Botao Xiao $^{6}$. Chuoji Huang ${ }^{2,3} \cdot$ Ruth L. Katz ${ }^{7}$. Chunxue Bai ${ }^{1}$ (])
}

Received: 26 October 2020 / Accepted: 10 January 2021 / Published online: 6 February 2021

(c) The Author(s) 2021

\begin{abstract}
Purpose Available biomarkers lack sensitivity for an early lung cancer. Circulating genetically abnormal cells (CACs) occur early in tumorigenesis. To determine the diagnostic value of CACs in blood detected by 4-color fluorescence in situ hybridization (FISH) for lung cancer.

Methods This was a prospective study of patients with pulmonary nodules $\leq 30 \mathrm{~mm}$ detected between 10/2019 and 01/2020 at four tertiary hospitals in China. All patients underwent a pathological examination of lung nodules found by imaging and were grouped as malignant and benign. CACs were detected by 4-color FISH. Patients were divided into the training and validation cohorts. Receiver operating characteristics analysis was used to analyze the diagnosis value of CACs.

Results A total of 205 participants were enrolled. Using a cut-off value of $\geq 3$, blood CACs achieved areas under the curve (AUCs) of $0.887,0.823$, and 0.823 for lung cancer in the training and validation cohorts, and all patients, respectively. CACs had high diagnostic values across all tumor sizes and imaging lesion types. CACs were decreased after surgery (median, 4 vs. $1, P<0.001$ ) in the validation set. The CAC status between blood and tissues was highly consistent (kappa $=0.909, P<0.001)$. The AUC of CAC (0.823) was higher than that of CEA (0.478), SCC (0.516), NSE (0.506), ProGRP (0.519), and CYFRA21-1 (0.535) (all $P<0.001)$. Conclusion CACs might have a high value for the early diagnosis of lung cancer. These findings might need to be validated in future studies. Evidence suggested homology in genetic aberrations between the CACs and the tumor cells.
\end{abstract}

Keywords Non-small cell lung cancer · Early detection of cancer · Early diagnosis · Circulating genetically abnormal cells · Fluorescence in situ hybridization

\section{Introduction}

Lung cancer is the leading cause of mortality globally, especially in smokers and the elderly (NCCN Clinical Practice Guidelines in Oncology (NCCN Guidelines). Non-Small

Mingxiang Feng, Xin Ye, Baishen Chen, Juncheng Zhang, Chuoji Huang and Chunxue Bai have contributed equally to this work.

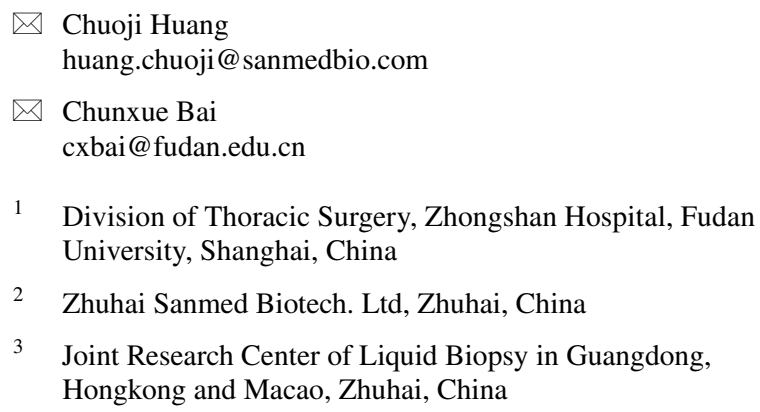

3 Joint Research Center of Liquid Biopsy in Guangdong, Hongkong and Macao, Zhuhai, China

Cell Lung Cancer 2020; Novello et al. 2016). There were an estimated 2,093,876 new cases of lung cancer worldwide in 2018, with an annual age-standardized incidence of 31.5/100,000 in men and 14.6/100,000 in women (Bray et al. 2018). The National Comprehensive Cancer Network (NCCN), the CHEST guideline and expert panel report, and the US Preventive Services Task Force recommended

4 Department of Thoracic surgery, Respiratory Center of Suining Central Hospital, An Affiliated Hospital of Chongqing Medical University, An Affiliated Hospital of North Sichuan Medical College, Suining, Sichuan, China

5 Department of Thoracic Surgery, Sun Yat-Sen Memorial Hospital of Sun Yat-Sen University, Guangzhou, China

6 School of Biology and Biological Engineering, South China University of Technology, Guangzhou, China

7 Department of Pathology, The University of Texas MD Anderson Cancer Center, Houston, TX, USA 
low-dose computed tomography (LDCT) screening for people aged 55-74, currently smoking or with $\geq 30$ pack-year history of smoking, and past smokers for $<15$ years (Mazzone et al. 2018; Moyer and Force 2014; NCCN Clinical Practice Guidelines in Oncology (NCCN Guidelines). Lung Cancer Screening 2019), and screening can be considered in individuals $\geq 50$ years of age, $a \geq 20$ pack-year history of smoking, and additional risk factors (including personal history of cancer or lung disease, family history of lung cancer, radon exposure, or relevant occupational exposure) that increases the risk of lung cancer to $\geq 1.3 \%$ (not including second-hand smoke exposure) (Mazzone et al. 2018; Moyer and Force 2014; NCCN Clinical Practice Guidelines in Oncology (NCCN Guidelines). Lung Cancer Screening. 2019). Early diagnosis is paramount for prognosis, since the five-year survival rate is $56 \%$ for patients with localized disease, $30 \%$ for those with regional disease, but only $5 \%$ for individuals distant disease (NCCN Clinical Practice Guidelines in Oncology (NCCN Guidelines). Lung Cancer Screening 2019; NCCN Clinical Practice Guidelines in Oncology (NCCN Guidelines). Non-Small Cell Lung Cancer 2020).

The current early detection methods for lung cancer are not sufficient. Indeed, available biomarkers (like carcinoembryonic antigen) and circulating nucleic acids have a low sensitivity (O'Flaherty et al. 2017; Plaks et al. 2013; Sorber et al. 2017). Plain X-ray, computed tomography (CT), and positron-emission tomography (PET)/CT have relatively high rates of false-positive and have low sensitivity for tumors $<10 \mathrm{~mm}$ and pure ground-glass nodules (National Lung Screening Trial Research et al. 2011; Wisnivesky et al. 2006; Yamagami et al. 2004; Yamauchi et al. 2011). Importantly, biopsy is an invasive diagnosis method and is associated with possible complications like infection and pneumothorax.

Genetic abnormalities in tumor suppressor genes and proto-oncogene are common in lung cancer (Hirsch et al. 2001; Zochbauer-Muller et al. 2002), but cannot be used for the screening of lung cancer because of the low yield of cancer cells in the blood, tumor heterogeneity, and unknown imminency between the detection of the abnormality and actual malignant transformation (Romeo et al. 2003). Cancer cells are often unable to maintain chromosome numbers and structures because of rapid uncontrolled growth and division (Romeo et al. 2003). Chromosomal aberrations, including rearrangements and aneusomy, are frequently found in early lung cancer (Haruki et al. 2001; Schenk et al. 1997; Taguchi et al. 1996). Importantly, a study of seven lung cancer specimens showed that chromosomal instability is found both in $8.5 \%$ and $59 \%$ of the premalignant and malignant lesions, respectively, within the same patients (Zojer et al. 2000), indicating that specific chromosomal aberrations occur in the early stage of tumorigenesis (Romeo et al. 2003).
Circulating genetically abnormal cells (CACs; i.e., cells that carry chromosomal instability) occur early in tumorigenesis and CACs can be detected from the blood (Katz et al. 2010, 2020). Katz et al. developed a 4-color fluorescence in situ hybridization (FISH) assay to identify CACs from peripheral blood of lung cancer patients using chromosomes 3 and 10 (probes for 3p22.1/3q29 (196F4) and 10q22.3/CEP10) (Katz et al. 2010). These abnormalities have previously been shown to commonly occur in lung cancer samples by the comparative genomic hybridization analysis (Jiang et al. 2004).

The study aimed to evaluate the diagnostic value of CACs detected by 4-color FISH for lung cancer, as well as to examine the genetic abnormalities between CACs and tumor cells. The results could be a novel sensitive and specific biomarker for the early detection of the disease.

\section{Materials and methods}

\section{Study design and participants}

This was a prospective study of patients with pulmonary nodules detected between October 2019 and January 2020 at the Zhongshan Hospital Affiliated to Fudan University, the Second Hospital Affiliated to Zhongshan University (Sun Yat-Sen Memorial Hospital), Suining Central Hospital, and Shanghai Chest Hospital. The study has been approved by the ethics committee of Zhongshan Hospital Affiliated to Fudan University (b2019-185r) and by the ethics review committees of the three other hospitals. Written inform consent was provided by all participants before the study.

The inclusion criteria were: (1) single or multiple pulmonary nodules $\leq 30 \mathrm{~mm}$ detected by CT or LDCT within the past 6 months; (2) $\geq 18$ years of age; and (3) planned to undergo non-surgical biopsy or surgical resection of the pulmonary nodules and histopathological examination. The exclusion criteria were: (1) lactating, pregnant, or preparing pregnant women; (2) severe heart, lung, liver, or kidney dysfunction or mental disorders; (3) previous clinical therapeutic interventions related to lung cancer, such as surgery, radiotherapy, chemotherapy, targeted treatment, or immunotherapy; (4) sampling problem that could not meet the requirements for histopathological examination; or (5) history of a malignant tumor within 5 years.

\section{Grouping}

CAC detection was performed for all participants within 5 days preoperatively, and within 5 days postoperatively for some of them. We enrolled the participants without 
postoperative blood collection as the training set and those with postoperative blood collection as the validation set.

\section{CAC detection}

Peripheral blood $(10 \mathrm{ml})$ and tissue samples were collected preoperatively and postoperatively for CAC detection. Chromosome 3 and 10 [probes for 3p22.1/3q29 (196F4) and 10q22.3/CEP10] abnormalities of peripheral blood mononuclear cells (PBMCs) of the pulmonary nodule population were qualitatively detected using the Mononuclear Cell Chromosomal Abnormality Detection Kit (Zhuhai Sanmed Biotechnology Ltd.). The assay was performed according to manufacturer's manual and was described in previous publications (cite our own papers). In brief, PBMCs were enriched via Ficoll density gradient and deposited to microscope glass slides by Cytospin system (Thermo Fisher). The cells were subsequently fixed for 4-color FISH (Katz et al. 2010, 2020; Yendamuri et al. 2008) or storage at $-20{ }^{\circ} \mathrm{C}$. Cell nuclei were stained with 4', 6-diamidino-2-phenylindole (DAPI).

The FISH samples were digitalized by the Duet System (Allegro plus, BioView Ltd.) to visualize the chromosomal targets in Chr. 3 and 10. Signal distribution in each cell was enumerated to identify chromosome loci gain or loss. Cells with polysomy in at least two different fluorescence channels were characterized as CACs.

\section{Biomarkers}

Peripheral blood samples were taken from each participant in 3-ml anticoagulant tubes for the measurement of the carcinoembryonic antigen (CEA), progastrin-releasing protein (ProGRP), squamous cell carcinoma antigen (SCC), and CYFRA21-1 levels. On the same day as collection, the tumor biomarkers were measured using electrochemiluminescence immunoassays (ECLIA) on a Roche Elecsys E170 analyzer (Roche Diagnostics, Switzerland).

\section{Data collection}

The characteristics of the participants (age, sex, and smoking history) were collected. The diagnosis of the nodules was classified into malignant (lung squamous cell carcinoma, invasive adenocarcinoma, micro-invasive adenocarcinoma, and malignant others) and benign (benign tumors, infection/inflammatory lesions, and benign others). The largest nodule size and the largest nodule type for each patient were recorded. LDCT examination was performed by the 64-section multidetector CT machine (Siemens, Erlangen, Germany).

\section{Statistical analysis}

Continuous data with a normal distribution were presented as means \pm standard deviation and analyzed using the independent sample $t$-test. Continuous data with a skewed distribution were presented as medians (interquartile ranges) and analyzed using the Mann-Whitney $U$ test. Categorical data were expressed as $n(\%)$ and analyzed using the chisquare test or Fisher's exact probability test. Receiver operating characteristic (ROC) curves were used to identify the cut-off value for CACs, the area under the curve (AUC), sensitivity, specificity, and other indicators. The numbers of postoperative and preoperative CACs in the validation set were tested using the Wilcoxon signed-rank sum test. The consistency of CACs in blood and tissue was tested by the Kappa test. All analyses were performed using SPSS 22.0 (IBM, Armonk, NY, USA). Two-sided $P$ values $<0.05$ were considered statistically significant.

\section{Results}

\section{Baseline information}

A total of 205 participants were enrolled (112 in the training set and 93 in the validation set) (Table 1). There were 97 (47.3\%) males. The median age was 62 (54-67) years. Among the 205 participants, 168 (82.0\%) had malignant lesions. The median longest diameter of the nodules was 18 (12-24) $\mathrm{mm}$. Seventy participants $(34.2 \%)$ had pure groundglass lesions, 96 (46.8\%) had solid lesions, and 39 (19.0\%) had mixed lesions. CACs were counted from the participants' blood before and after the operation; the median preoperative and postoperative CAC counts were $4(2-7)$ and $1(0-5)$, respectively. There were no significant differences between the two sets (all $\mathrm{P}>0.05$ ).

\section{ROC analysis of CACs for the diagnosis of lung cancer}

Figure 1 shows a typical case of a patient with a large solid nodule in the lung and positive CAC results. Preoperative CT revealed a solid nodule (Fig. 1a). CACs were analyzed by 4-color fluorescence in situ hybridization (Fig. 1b). The CEP10 is diploid and it has a split/diffused signal. The combined images of CACs show polysomy/gain of 3p22.1 (red), polysomy/gain of 3q29 (green), whereas 10q22.3 (gold, two copies) and CEP10 (aqua) was diploid (Fig. 1c). The participant had 3 CACs before surgery and 0 after surgery (Fig. 1d). Histopathological examination revealed adenocarcinoma of the lung. The chromosomal abnormalities were also found in the tissue specimens (Fig. 1e). 
Table 1 Baseline characteristics of the patients

\begin{tabular}{|c|c|c|c|c|}
\hline & Total $n=205$ & Training set $n=112$ & Validation set $n=93$ & $P$ \\
\hline Sex, $n(\%)$ & & & & 0.573 \\
\hline Male & $97(47.3 \%)$ & $55(49.1 \%)$ & $42(45.2 \%)$ & \\
\hline Female & $108(52.7 \%)$ & $57(50.9 \%)$ & $51(54.8 \%)$ & \\
\hline Age, median (IQR) & $62(54,67)$ & $62(55,68)$ & $62(52,66)$ & 0.246 \\
\hline Smoking history, $n(\%)$ & $51(24.9 \%)$ & $28(25.0 \%)$ & $23(24.7 \%)$ & 0.965 \\
\hline Benign and malignant, $n(\%)$ & & & & 0.310 \\
\hline Benign & $37(18.1 \%)$ & $23(20.5 \%)$ & $14(15.1 \%)$ & 0.692 \\
\hline Benign tumor & $11(5.4 \%)$ & $8(7.1 \%)$ & $3(3.2 \%)$ & \\
\hline Infection/inflammatory lesions & $22(10.7 \%)$ & $13(11.6 \%)$ & $9(9.7 \%)$ & \\
\hline Other & $4(2.0 \%)$ & $2(1.8 \%)$ & $2(2.2 \%)$ & \\
\hline Malignant & $168(82.0 \%)$ & $89(79.5 \%)$ & $79(85.0 \%)$ & 0.113 \\
\hline Squamous cell carcinoma of lung & $8(3.9 \%)$ & $2(1.8 \%)$ & $6(6.5 \%)$ & \\
\hline Invasive adenocarcinoma & $84(41.0 \%)$ & $44(39.3 \%)$ & $40(43.0 \%)$ & \\
\hline Microinvasive adenocarcinoma & $72(35.1 \%)$ & $39(34.8 \%)$ & $33(35.5 \%)$ & \\
\hline Other & $4(2.0 \%)$ & $4(3.6 \%)$ & 0 & \\
\hline Maximum nodule size, median (IQR) & $18(12,24)$ & $18(13,23)$ & $19(11,25)$ & 0.560 \\
\hline Maximum nodule type, n (\%) & & & & 0.074 \\
\hline Pure ground glass type & $70(34.2 \%)$ & $42(37.5 \%)$ & $28(30.1 \%)$ & \\
\hline Mixed & $39(19.0 \%)$ & $15(13.4 \%)$ & $24(25.8 \%)$ & \\
\hline Solid & $96(46.8 \%)$ & $55(49.1 \%)$ & $41(44.1 \%)$ & \\
\hline Preoperative CAC, median (IQR) & $4(2,7)$ & $4(2,6)$ & $4(3,7)$ & 0.893 \\
\hline Postoperative CAC, median (IQR) & $1(0,5)$ & NA & $1(0,5)$ & NA \\
\hline
\end{tabular}

$I Q R$ interquartile range, $C A C$ genetically abnormal cells
We achieved an $86.5 \%$ sensitivity rate, a $78.3 \%$ specificity, an AUC of 0.887 , a $93.9 \%$ positive predictive value (PPV), a $60.0 \%$ negative predictive value (NPV), and the 3.98 positive likelihood ratio (PLR) in the training set, when the cut-off value is set as $\geq 3$ CAC (Fig. 2). In the validation set, using the same CAC cut-off value, we observed $86.1 \%$ sensitivity, 78.6\% specificity, 0.823 AUC, $95.8 \%$ PPV, 50.0\% NPV, and 4.02 PLR (Fig. 2b). In all participants, using a cut-off value of $\geq 3$ CACs, $86.3 \%$ sensitivity, $78.4 \%$ specificity, 0.823 AUC, 94.8\% PPV, 55.8\% NPV, and 3.99 PLR were obtained (Fig. 2c). Supplementary Table 1 shows the sensitivity and specificity values according to different CAC cut-off levels. It should be noted that the three data sets of specimens yielded similar results, suggesting that the training and validation sets were consistent.

\section{CAC diagnostic power in different nodule sizes}

The participants were divided according to their lesion size. In participants with a lesion of $0-9 \mathrm{~mm}$, using a cut-off value of $\geq 3$, CAC achieved $84.2 \%$ sensitivity, $85.7 \%$ specificity, AUC of $0.850,94.1 \%$ PPV, 66.7\% NPV, and 5.90 PLR (Supplementary Fig. 1a). In participants with a lesion of 10-29 mm, CAC achieved $85.5 \%$ sensitivity, $74.1 \%$ specificity, AUC of $0.798,94.4 \%$ PPV, 50.0\% NPV, and 3.30 PLR (Supplementary Fig. 1b). In participants with a lesion of
$30 \mathrm{~mm}$, CAC achieved 100\% sensitivity, 100\% specificity, AUC of 1.000, 100\% PPV, 100\% NPV, and not-applicable PLR (Supplementary Fig. 1c). Those results indicate that the tumor size has minimal or no impact on the diagnostic value of CACs.

\section{CAC diagnostic power in different nodule types}

The participants were grouped according to the type of lung lesion at imaging. In participants with a pure ground glass, solid, and mixed lesions and using a cut-off value of $\geq 3$, CAC achieved $82.0 \% / 90.4 \% / 85.3 \%$ sensitivity, $77.8 \% / 73.9 \% / 100 \%$ specificity, AUC of 0.799/0.822/0.926, 96.2\%/91.7\%/100\% PPV, 38.9\%/70.8\%/50.0\% NPV, and 3.69/3.47/NA PLR (Supplementary Fig. 2). Those results indicate that the type of radiological lesion has no impact on the diagnostic value of CACs.

\section{Comparison of CACs in patients with single or multiple nodules}

26 subjects had multiple nodules and the other 179 had a single nodule. The median number of CACs in patients with single nodule was similar to that of patients with multiple nodules (4 vs. 4). 
A
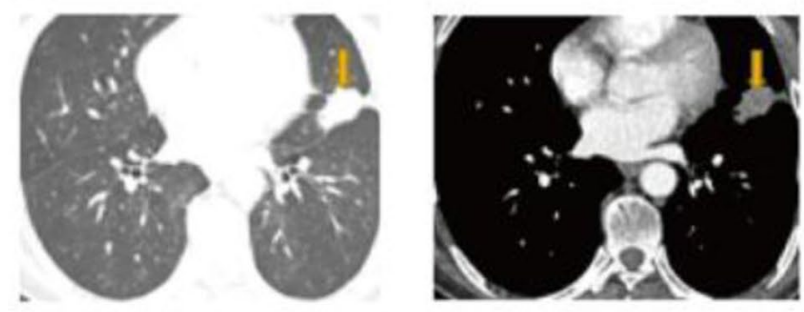

C

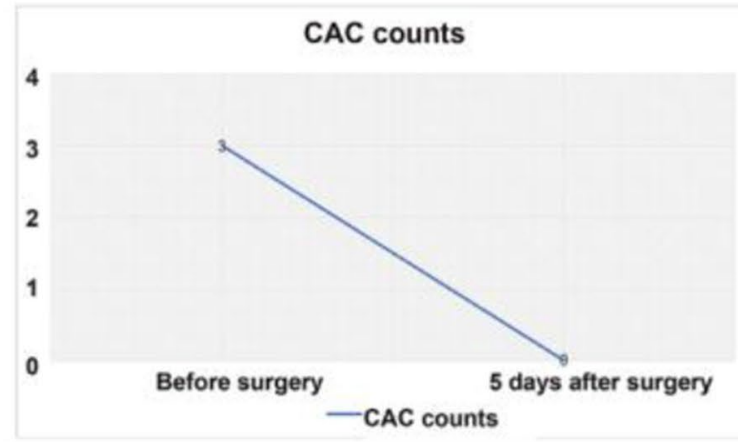

D

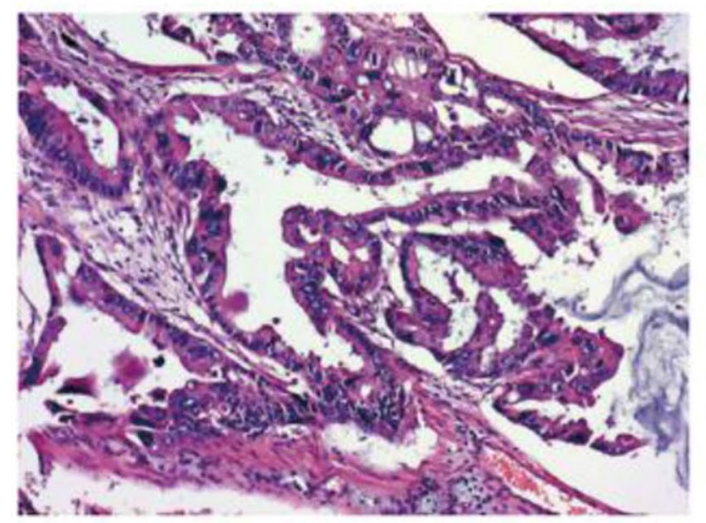

Fig. 1 A typical case. a Preoperative computed tomography scans. b CAC schematic diagram. Cells with 4', 6-diamidino-2-phenylindole (DAPI) staining (original magnification $\times 100$ ). The combined images of CACs show the polysomy/gain of 3p22.1 (red), polysomy/gain of 3q29 (green), and CEP10 (aqua), whereas 10q22.3 (gold, two copies) was diploid. Genetic abnormalities were identified using a 4-color cocktail of FISH probes on a BioView Duet-3 instrument (original

\section{Correlation between blood and corresponding lung cancer tissue}

78 patients' peripheral blood and tumor tissues, who underwent surgical resection of their lung tumors, were obtained in paired sets. The same set of 4-color FISH probes was used in both the blood and tumor specimens. We observed

\section{B}

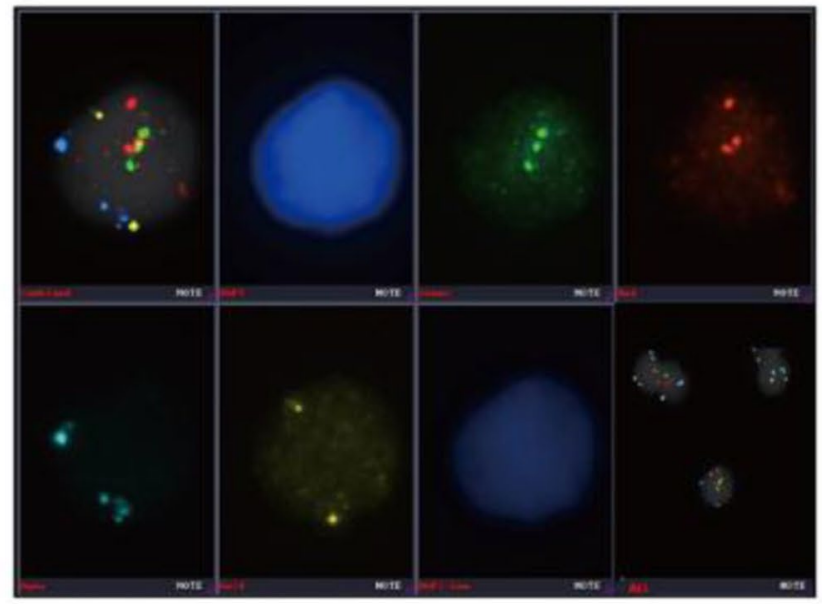

E

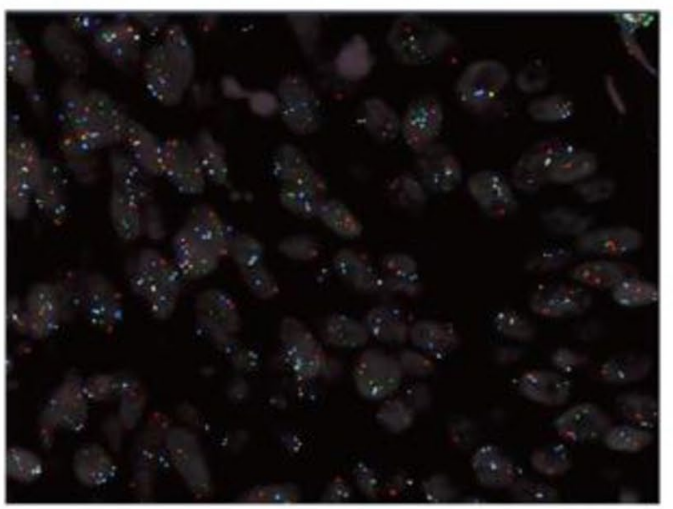

magnification $\times 400$ ): three red signals consistent with three copies of 3 p22.1; three aqua signals representing three copies of CEP10; three green signals representing three copies of 3q29; and two gold signals consistent with two copies of 10q22.3. $\mathbf{c}$ Three CACs were found by a 4-color cocktail of FISH probes on a BioView Duet-3 instrument. d Pathological HE revealed adenocarcinoma of the lung. e Chromosomal abnormalities in the tissue

a significant correlation between four genetical abnormalities in PBMCs and corresponding biomarkers in the tumor specimens. Table 2 shows the postoperative and preoperative CAC counts in the validation set. The CAC count was significantly decreased after the surgery (median, 4 vs. 1 , $P<0.001)$. Table 3 shows that the genetic abnormalities 
A

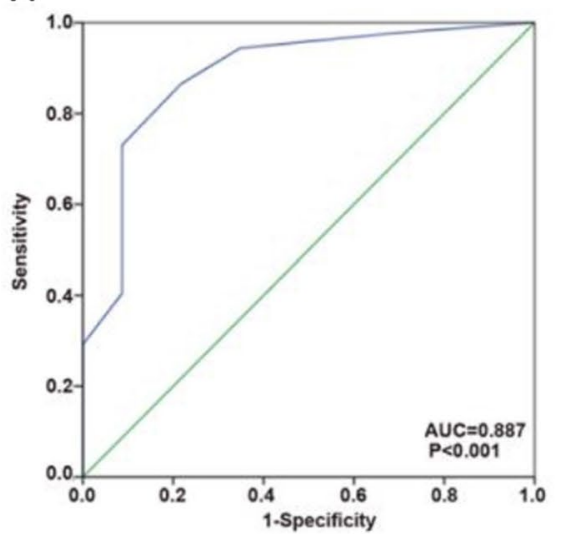

B

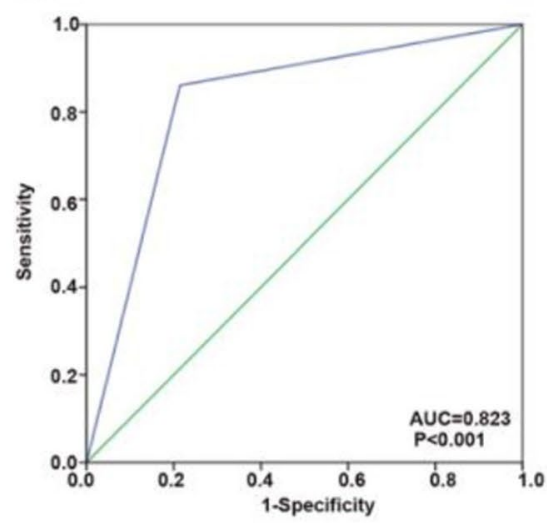

C

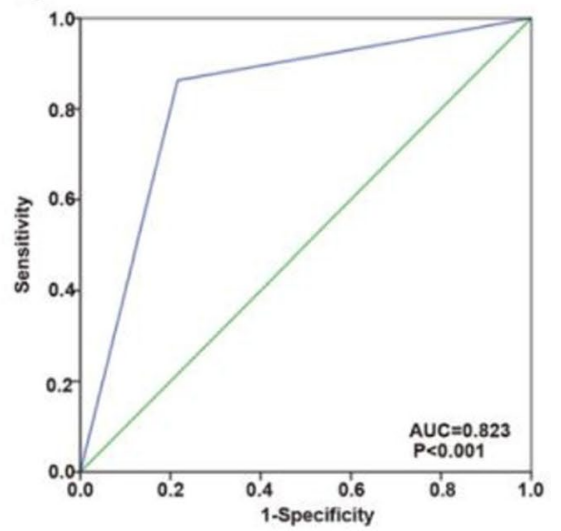

Fig. 2 Receiver operating characteristics analysis of genetically abnormal cells (CAC) for non-small cell lung cancer. a Training set. Using a cut-off value of $\geq 3$ CAC achieved $86.5 \%$ sensitivity, $78.3 \%$ specificity, the area under the curve (AUC) of $0.887,93.9 \%$ positive predictive value (PPV), 60.0\% negative predictive value (NPV), and

Table 2 Comparison of the numbers of preoperative and postoperative CACs

\begin{tabular}{llll}
\hline & Preoperative CAC & Postoperative CAC & $P$ \\
\hline Median $(\mathrm{IQR})$ & $4(3,7)$ & $1(0,5)$ & $<0.001$ \\
\hline
\end{tabular}

$I Q R$ interquartile range, $C A C$ genetically abnormal cells
3.98 positive likelihood-ratio (PLR). b Validation set. Using a cutoff value of $\geq 3$ CAC achieved $86.1 \%$ sensitivity, $78.6 \%$ specificity, AUC of $0.823,95.8 \%$ PPV, 50.0\% NPV, and 4.02 PLR. c All patients. Using a cut-off value of $\geq 3$ CAC achieved $86.3 \%$ sensitivity, $78.4 \%$ specificity, AUC of $0.823,94.8 \%$ PPV, $55.8 \%$ NPV, and 3.99 PLR

between CACs and tumor cells were highly consistent (kappa $=0.909, P<0.001)$.

Of the 78 subjects, 63 were diagnosed with malignant tissues in histopathological examinations. Of these 63 subjects, $52(82.54 \%)$ had chromosome abnormalities detected in the tissue test, while none of the 15 subjects with benign tissues had chromosome abnormalities in the tissue test.
Table 3 Comparison in chromosomal abnormalities in blood and tissue

\begin{tabular}{lllll}
\hline & $\begin{array}{l}\text { Positive tissue chro- } \\
\text { mosome }\end{array}$ & $\begin{array}{l}\text { Negative tissue chro- } \\
\text { mosome }\end{array}$ & Kappa & $P$ \\
\hline Blood positive $(\mathrm{CAC} \geq 3)$ & 53 & 3 & 0.909 & $<0.001$ \\
Blood negative $(\mathrm{CAC}<3)$ & 0 & 22 & & \\
\hline
\end{tabular}

CAC genetically abnormal cells

Table 4 Sensitivity and specificity of common biomarkers

\begin{tabular}{llrllll}
\hline Biomarker & AUC & $P$ & $95 \%$ CI & Sensitivity & Specificity & $\begin{array}{l}\text { Comparison } \\
\text { with AUC of } \\
\text { CAC }\end{array}$ \\
\hline CEA & 0.478 & 0.684 & $(0.372,0.585)$ & 0.151 & 0.806 & $<0.001$ \\
SCC & 0.516 & 0.775 & $(0.408,0.624)$ & 0.032 & 1.000 & $<0.001$ \\
NSE & 0.506 & 0.915 & $(0.399,0.613)$ & 0.071 & 0.941 & $<0.001$ \\
Pro-GRP & 0.519 & 0.794 & $(0.383,0.654)$ & 0.037 & 1.000 & $<0.001$ \\
CYFRA21-1 & 0.535 & 0.522 & $(0.432,0.637)$ & 0.184 & 0.886 & $<0.001$ \\
CAC & 0.823 & $<0.001$ & $(0.741,0.906)$ & 0.863 & 0.784 & \\
\hline
\end{tabular}

$A U C$ area under the curve, $C I$ confidence interval, $I Q R$ interquartile range, $C A C$ genetically abnormal cells, $C E A$ carcinoembryonic antigen, $S C C$ squamous cell carcinoma antigen, NSE neuron-specific enolase, pro$G R P$ pro-gastrin releasing peptide 


\section{Sensitivity and specificity of common biomarkers}

The diagnostic value of different biomarkers was assessed and compared with CACs (Table 4). The AUC of CAC $(0.823)$ was higher than that of CEA (0.478), SCC (0.516), NSE (0.506), ProGRP (0.519), and CYFRA21-1 (0.535) (all $P<0.001)$.

\section{Discussion}

It has been well-documented that the commonly available serum tumor biomarkers provide a little diagnostic value for early lung cancer diagnosis (National Lung Screening Trial Research et al. 2011; O'Flaherty et al. 2017; Plaks et al. 2013; Sorber et al. 2017; Wisnivesky et al. 2006; Yamagami et al. 2004; Yamauchi et al. 2011). CACs occur early in tumorigenesis and might be of use as a biomarker for lung cancer (Katz et al. 2010, 2020). The results of this study indicate that CACs have a high value for the early diagnosis of lung cancer. This will have to be validated in future studies as an early screening tool for lung cancer. Nevertheless, the results could be a novel sensitive and specific biomarker for the early detection of the disease. In many studies on the early diagnosis of lung cancer, the control group includes healthy people (without nodules). The disadvantage of this control group is the lack of histopathological results, and the actual absence of lung cancer cannot be confirmed. Therefore, in the present study, the control group included patients with benign lung nodules proven pathologically.

In this study, CACs show a high diagnostic value for tumors of all sizes, even for tumors of $0-10 \mathrm{~mm}$. This is of particular interest in a screening context because PETCT has low sensitivity for tumors of $0-10 \mathrm{~mm}$ (sensitivity of $50 \%$ for lesions $<10 \mathrm{~mm}$ or $17 \%$ for lesions $<8 \mathrm{~mm}$ ) (Evangelista et al. 2012; Kim et al. 2007), and PET-CT is not indicated for lesions $<8 \mathrm{~mm}$ or $<10 \mathrm{~mm}$ (Cancer du poumon, Bilan initial, Collection Recommandations et référentiels 2011; Patel et al. 2013a, b). In the case of misdiagnosis, even though the patient could be followed up and get diagnosed in the next scanning, he/she could miss the best treatment window and allow the tumor to further develop and metastasize (NCCN Clinical Practice Guidelines in Oncology (NCCN Guidelines); Lung Cancer Screening 2019; NCCN Clinical Practice Guidelines in Oncology (NCCN Guidelines). Non-Small Cell Lung Cancer 2020). However, while other studies have suggested that the CAC number might increase as the tumor progresses and be related to tumor prognosis (Katz et al. 2020), we did not find a relationship with tumor size in this study. This could be because all the patients had tumors of less than $30 \mathrm{~mm}$. Further study of the patients with a longer follow-up might show the CAC numbers increase if the disease progresses. The type of lesion had a minimal influence on the diagnostic value. This study is the first to analyze the diagnostic value of CACs for lung cancer across lesion size, imaging lesion type, and comparing its performance with common tumor biomarkers. The results showed high AUCs for all included lesions $\leq 30 \mathrm{~mm}$, which are the lesions commonly found during early screening and considered benign (NCCN Clinical Practice Guidelines in Oncology (NCCN Guidelines). Lung Cancer Screening 2019).

This method of investigating CACs has some advantages over conventional chromosome testing of cells, which is performed by FISH-staining the tissues. Staining cells with chromosome abnormalities in peripheral blood involves liquid biopsy, which enables multiple, repeated and non-invasive evaluation of chromosome abnormalities in patients. Moreover, our system enables the automatic recognition and automatic counting of blood cells with chromosome abnormalities, which reduces the impact of observer bias. We also showed that the genetic abnormalities in the CACs were similar to those found in their respective tumors. This is supported by previous studies, in which CACs had similar characteristics to those of the primary tumor cells (Katz et al. 2010; Pailler et al. 2015). This homology might be an indication that the CACs found in peripheral blood might be tumor cells that detached themselves from the primary tumor and entered circulation in order to seed novel metastases. Besides, after the surgery, the CACs were significantly reduced, which supports the view that there was homology in genetic aberrations between the CACs and the tumor cells.

The numbers of CACs might very well represent the lung lesion and could be used for the early diagnosis of lung cancer. Indeed, more aggressive tumors will show less cell cohesion, leading to more CACs with the potential to seed metastases (Yendamuri et al. 2008). Of note, CACs can be found in premalignant lesions, and malignant lesions since the appearance of cytogenetic abnormalities that occur early in tumorigenesis (Zojer et al. 2000), highlighting their value for the early detection of lung cancer.

Multiple biomarkers in blood are used for tumor liquid biopsies, yet none has been extensively validated and utilized in clinical settings (O'Flaherty et al. 2017; Plaks et al. 2013; Sorber et al. 2017). The sensitivity and specificity of ctDNA for early lung cancer were $53.8 \%$ and $47.3 \%$, respectively (Chen et al. 2016). Regarding CTCs detected using the Veridex CellSearch system, which is based on an antiEpCAM antibody, the sensitivity for early lung cancer was only $19.3 \%$ (Tanaka et al. 2009). In comparison, the present study showed a sensitivity of $86 \%$ to detect lung cancer in all participants. Nevertheless, this conclusion must be taken with caution as those different biomarkers were not assessed head-to-head in the same patients. Future studies should 
include CACs, CTCs, and tumor DNA, and those should be tested in healthy controls for the validation to ensure that those tests are negative in patients without cancer.

More traditional biomarkers like CEA, ProGRP, SCC, NSE, and CYFRA21-1 are commonly used for the diagnosis and management indicators of lung cancer (Mishra and Verma 2010; NCCN Clinical Practice Guidelines in Oncology (NCCN Guidelines). Non-Small Cell Lung Cancer 2020; Novello et al. 2016), but they are not designed for the early screening of lung cancer due to low sensitivity (NCCN Clinical Practice Guidelines in Oncology (NCCN Guidelines). Lung Cancer Screening 2019; Neal et al. 2015; Vargas and Harris 2016). In the present study, the AUC and sensitivity of CACs for early lung cancer were higher than any of those five biomarkers, suggesting that CAC could be a sensitive marker for lung cancer early diagnosis working in conjunction with LDCT.

Despite its strengths, there are several limitations in this study. For instance, we only assessed a relatively small cohort with high disease prevalence, which may not be sufficient to establish reliable correlations between CACs and the clinicopathological characteristics of the patients. Nevertheless, a strength of the present study is that all participants had a pathological diagnosis, even the benign nodules. Many previous studies included patients with tuberculosis or chronic inflammation, and they rarely included those with benign tumors and granuloma. Additional studies with larger cohorts would be needed to guarantee the robustness of the ROC analysis and identify most powerful threshold of CACs for lung cancer early diagnosis. Another limitation was that other popular liquid biopsies biomarkers, such as ctDNA and CTC, were not parallelly analyzed. Notwithstanding these limitations, the study findings underscore an interesting biological process during lung cancerogenesis and identification of a novel biomarker for lung cancer early diagnosis.

In conclusion, CACs could be a promising biomarker for the early diagnosis of lung cancer. In a screening context, implementing such a diagnostic tool may benefit lung cancer patients with detection at an early stage and improve prognosis. Our study also suggests that there may be a high homology in genetic abnormalities between the CACs and the tumor cells in cancer tissue.

\section{Abbreviations}

NCCN: National Comprehensive Cancer Network; LDCT: Low-dose computed tomography; CT: Computed tomography; PET: Positron-emission tomography; CACs: Circulating genetically abnormal cells; FISH: Fluorescence in situ hybridization; CEA: Carcinoembryonic antigen; ProGRP: Progastrin-releasing protein; SCC: Squamous cell carcinoma antigen; ECLIA: Electrochemiluminescence immunoassays; ROC: Receiver operating characteristic; AUC: Area under the curve
Supplementary Information The online version contains supplementary material available at https://doi.org/10.1007/s00432-021-03517-6.

Acknowledgements The authors acknowledged the help of the medical team at Zhuhai SanMed Biotech Ltd. for their study design work and MDA test services for CACs detection.

Author contributions $\mathrm{MF}, \mathrm{XY}, \mathrm{CH}, \mathrm{JZ}$ and $\mathrm{CB}$ have contributed equally in carrying out the studies. ML, $\mathrm{HZ}, \mathrm{BX}$ and $\mathrm{BC}$ performed the statistical analysis and participated in its design. $\mathrm{MH}, \mathrm{YC}$ and $\mathrm{YZ}$ helped to draft the manuscript. All authors read and approved the final manuscript.

Funding This study was funded by the program for Guangdong Introducing Innovative and Entrepreneurial Teams (2019ZT08Y297).

Availability of data and materials The datasets used and/or analyzed during the current study are available from the corresponding author on reasonable request.

\section{Compliance with ethical standards}

Conflict of interest The authors declare that they have no competing interests.

Ethics approval The study has been approved by the ethics committee of Zhongshan Hospital Affiliated to Fudan University (b2019-185r) and by the ethics review committees of the three other hospitals. Written inform consent was provided by all participants before the study.

Open Access This article is licensed under a Creative Commons Attribution 4.0 International License, which permits use, sharing, adaptation, distribution and reproduction in any medium or format, as long as you give appropriate credit to the original author(s) and the source, provide a link to the Creative Commons licence, and indicate if changes were made. The images or other third party material in this article are included in the article's Creative Commons licence, unless indicated otherwise in a credit line to the material. If material is not included in the article's Creative Commons licence and your intended use is not permitted by statutory regulation or exceeds the permitted use, you will need to obtain permission directly from the copyright holder. To view a copy of this licence, visit http://creativecommons.org/licenses/by/4.0/.

\section{References}

Bray F, Ferlay J, Soerjomataram I, Siegel RL, Torre LA, Jemal A (2018) Global cancer statistics 2018: GLOBOCAN estimates of incidence and mortality worldwide for 36 cancers in 185 countries CA Cancer J Clin 68:394-424 doi:https://doi.org/10.3322/ caac. 21492

Cancer du poumon, Bilan initial, Collection Recommandations et référentiels (2011). INCa, Boulogne-Billancourt

Chen KZ et al (2016) Circulating tumor DNA detection in early-stage non-small cell lung cancer patients by targeted sequencing. Sci Rep 6:31985. https://doi.org/10.1038/srep31985

Evangelista et al (2012) Indeterminate pulmonary nodules on CT images in breast cancer patient: the additional value of $18 \mathrm{~F}-\mathrm{FDG}$ PET/CT. J Med Imaging Radiat Oncol 56:417-424 doi: https:// doi.org/10.1111/j.1754-9485.2012.02408.x 
Haruki et al (2001) Persistent increase in chromosome instability in lung cancer: possible indirect involvement of p53 inactivation. Am J Pathol 159:1345-1352 doi:https://doi.org/10.1016/S0002 -9440(10)62521-7

Hirsch FR, Franklin WA, Gazdar AF, Bunn PA Jr (2001) Early detection of lung cancer: clinical perspectives of recent advances in biology and radiology. Clin Cancer Res 7:5-22

Jiang F, Yin Z, Caraway NP, Li R, Katz RL (2004) Genomic profiles in stage I primary non small cell lung cancer using comparative genomic hybridization analysis of cDNA microarrays. Neoplasia 6:623-635. https://doi.org/10.1593/neo.04142

Katz et al (2020) Identification of circulating tumor cells using 4-color fluorescence in situ hybridization: Validation of a noninvasive aid for ruling out lung cancer in patients with low-dose computed tomography-detected lung nodules. Cancer Cytopathol doi:https ://doi.org/10.1002/cncy.22278

Katz RL et al (2010) Genetically abnormal circulating cells in lung cancer patients: an antigen-independent fluorescence in situ hybridization-based case-control study. Clin Cancer Res 16:3976-3987. https://doi.org/10.1158/1078-0432.CCR-09-3358

Kim SK et al (2007) Accuracy of PET/CT in characterization of solitary pulmonary lesions. J Nucl Med 48:214-220

Mazzone PJ et al (2018) Screening for lung cancer: CHEST guideline and expert panel report. Chest 153:954-985. https://doi. org/10.1016/j.chest.2018.01.016

Mishra A, Verma M (2010) Cancer biomarkers: are we ready for the prime time? Cancers (Basel) 2:190-208. https://doi.org/10.3390/ cancers 2010190

Moyer VA, Force USPST (2014) Screening for lung cancer: US Preventive Services Task Force recommendation statement. Ann Intern Med 160:330-338. https://doi.org/10.7326/M13-2771

National Lung Screening Trial Research T et al (2011) Reduced lungcancer mortality with low-dose computed tomographic screening. N Engl J Med 365:395-409 doi:https://doi.org/10.1056/NEJMo a1102873

NCCN Clinical Practice Guidelines in Oncology (NCCN Guidelines). Lung Cancer Screening. (2019). Version 1.2020 edn. National Comprehensive Cancer Network, Washington

NCCN Clinical Practice Guidelines in Oncology (NCCN Guidelines). Non-Small Cell Lung Cancer. (2020). Version 4.2020 edn. National Comprehensive Cancer Network, Washington

Neal JW, Gainor JF, Shaw AT (2015) Developing biomarker-specific end points in lung cancer clinical trials. Nat Rev Clin Oncol 12:135-146. https://doi.org/10.1038/nrclinonc.2014.222

Novello et al (2016) Metastatic non-small-cell lung cancer: ESMO Clinical Practice Guidelines for diagnosis, treatment and followup. Ann Oncol 27:v1-v27 doi:https://doi.org/10.1093/annonc/ mdw326

O'Flaherty L, Wikman H, Pantel K (2017) Biology and clinical significance of circulating tumor cell subpopulations in lung cancer. Transl Lung Cancer Res 6:431-443. https://doi.org/10.21037/ tlcr.2017.07.03

Pailler et al (2015) High level of chromosomal instability in circulating tumor cells of ROS1-rearranged non-small-cell lung cancer. Ann Oncol 26:1408-1415 doi:https://doi.org/10.1093/annonc/mdv165

Patel et al (2013a) A practical algorithmic approach to the diagnosis and management of solitary pulmonary nodules: part 1: radiologic characteristics and imaging modalities. Chest 143:825-839 doi:https://doi.org/10.1378/chest.12-0960

Patel et al (2013b) A practical algorithmic approach to the diagnosis and management of solitary pulmonary nodules: part 2: pretest probability and algorithm. Chest 143:840-846 doi:https://doi. org/10.1378/chest.12-1487

Plaks V, Koopman CD, Werb Z (2013) Cancer. Circulating tumor cells. Science 341:1186-1188. https://doi.org/10.1126/science.1235226

Romeo MS et al (2003) Chromosomal abnormalities in non-small cell lung carcinomas and in bronchial epithelia of high-risk smokers detected by multi-target interphase fluorescence in situ hybridization. J Mol Diagn 5:103-112. https://doi.org/10.1016/s1525 -1578(10)60459-X

Schenk T, Ackermann J, Brunner C, Schenk P, Zojer N, Roka S, Drach J (1997) Detection of chromosomal aneuploidy by interphase fluorescence in situ hybridization in bronchoscopically gained cells from lung cancer patients. Chest 111:1691-1696. https://doi. org/10.1378/chest.111.6.1691

Sorber L et al (2017) Circulating cell-free nucleic acids and platelets as a liquid biopsy in the provision of personalized therapy for lung cancer patients. Lung Cancer 107:100-107. https://doi. org/10.1016/j.lungcan.2016.04.026

Taguchi T, Zhou JY, Feder M, Litwin S, Klein-Szanto AJ, Testa JR (1996) Detection of aneuploidy in interphase nuclei from nonsmall cell lung carcinomas by fluorescence in situ hybridization using chromosome-specific repetitive DNA probes. Cancer Genet Cytogenet 89:120-125. https://doi.org/10.1016/01654608(95)00355-x

Tanaka F et al (2009) Circulating tumor cell as a diagnostic marker in primary lung cancer. Clin Cancer Res 15:6980-6986. https://doi. org/10.1158/1078-0432.CCR-09-1095

Vargas AJ, Harris CC (2016) Biomarker development in the precision medicine era: lung cancer as a case study. Nat Rev Cancer 16:525-537. https://doi.org/10.1038/nrc.2016.56

Wisnivesky JP, Henschke CI, Yankelevitz DF (2006) Diagnostic percutaneous transthoracic needle biopsy does not affect survival in stage I lung cancer. Am J Respir Crit Care Med 174:684-688. https://doi.org/10.1164/rccm.200602-1600C

Yamagami T, Kato T, Iida S, Hirota T, Nishimura T (2004) Percutaneous needle biopsy for small lung nodules beneath the rib under CT scan fluoroscopic guidance with gantry tilt. Chest 126:744-747. https://doi.org/10.1378/chest.126.3.744

Yamauchi et al. (2011) Diagnostic performance of percutaneous coreneedle lung biopsy under CT scan fluoroscopic guidance for pulmonary lesions measuring $</=10 \mathrm{~mm}$. Chest $140: 1669-1670$ doi:https://doi.org/10.1378/chest.11-1821

Yendamuri et al. (2008) 3p22.1 and 10q22.3 deletions detected by fluorescence in situ hybridization (FISH): a potential new tool for early detection of non-small cell lung. Cancer (NSCLC) J Thorac Oncol 3:979-984 doi:https://doi.org/10.1097/JTO.0b013e3181 $834 \mathrm{f} 3 \mathrm{a}$

Zochbauer-Muller S, Gazdar AF, Minna JD (2002) Molecular pathogenesis of lung cancer. Annu Rev Physiol 64:681-708. https://doi. org/10.1146/annurev.physiol.64.081501.155828

Zojer N, Dekan G, Ackermann J, Fiegl M, Kaufmann H, Drach J, Huber H (2000) Aneuploidy of chromosome 7 can be detected in invasive lung cancer and associated premalignant lesions of the lung by fluorescence in situ hybridisation. Lung Cancer 28:225235 doi:https://doi.org/10.1016/s0169-5002(00)00097-0

Publisher's Note Springer Nature remains neutral with regard to jurisdictional claims in published maps and institutional affiliations. 